\title{
Mentalization for Offending Adult Males (MOAM): study protocol for a randomized controlled trial to evaluate mentalization- based treatment for antisocial personality disorder in male offenders on community probation
}

Peter Fonagy ${ }^{1,2^{*}} \mathbb{D}$, Jessica Yakeley ${ }^{3}$, Tessa Gardner ${ }^{2}$, Elizabeth Simes ${ }^{1,2}$, Mary McMurran $^{4}$, Paul Moran ${ }^{5}$, Mike Crawford ${ }^{6}$, Alison Frater ${ }^{7}$, Barbara Barrett ${ }^{8}$, Angus Cameron ${ }^{9}$, James Wason ${ }^{10,11}$, Stephen Pilling ${ }^{1}$, Stephen Butler ${ }^{12}$ and Anthony Bateman ${ }^{1,2}$

\begin{abstract}
Background: Antisocial personality disorder (ASPD), although associated with very significant health and social burden, is an under-researched mental disorder for which clinically effective and cost-effective treatment methods are urgently needed. No intervention has been established for prevention or as the treatment of choice for this disorder. Mentalization-based treatment (MBT) is a psychotherapeutic treatment that has shown some promising preliminary results for reducing personality disorder symptomatology by specifically targeting the ability to recognize and understand the mental states of oneself and others, an ability that is compromised in people with ASPD. This paper describes the protocol of a multi-site RCT designed to test the effectiveness and cost-effectiveness of MBT for reducing aggression and alleviating the wider symptoms of ASPD in male offenders subject to probation supervision who fulfil diagnostic criteria for ASPD.

(Continued on next page)
\end{abstract}

\footnotetext{
* Correspondence: p.fonagy@ucl.ac.uk

'Research Department of Clinical, Educational and Health Psychology,

University College London, London, UK

${ }^{2}$ Anna Freud National Centre for Children and Families, London, UK

Full list of author information is available at the end of the article
}

(c) The Author(s). 2020 Open Access This article is licensed under a Creative Commons Attribution 4.0 International License, which permits use, sharing, adaptation, distribution and reproduction in any medium or format, as long as you give appropriate credit to the original author(s) and the source, provide a link to the Creative Commons licence, and indicate if changes were made. The images or other third party material in this article are included in the article's Creative Commons licence, unless indicated otherwise in a credit line to the material. If material is not included in the article's Creative Commons licence and your intended use is not permitted by statutory regulation or exceeds the permitted use, you will need to obtain permission directly from the copyright holder. To view a copy of this licence, visit http://creativecommons.org/licenses/by/4.0/. The Creative Commons Public Domain Dedication waiver (http://creativecommons.org/publicdomain/zero/1.0/) applies to the data made available in this article, unless otherwise stated in a credit line to the data. 


\begin{abstract}
(Continued from previous page)
Methods: Three hundred and two participants recruited from a pool of offenders subject to statutory supervision by the National Probation Service at 13 sites across the UK will be randomized on a 1:1 basis to 12 months of probation plus MBT or standard probation as usual, with follow-up to 24 months post-randomization. The primary outcome is frequency of aggressive antisocial behaviour as assessed by the Overt Aggression Scale - Modified. Secondary outcomes include violence, offending rates, alcohol use, drug use, mental health status, quality of life, and total service use costs. Data will be gathered from police and criminal justice databases, NHS record linkage, and interviews and self-report measures administered to participants. Primary analysis will be on an intent-to-treat basis; per-protocol analysis will be undertaken as secondary analysis. The primary outcome will be analysed using hierarchical mixedeffects linear regression. Secondary outcomes will be analysed using mixed-effects linear regression, mixed-effects logistic regression, and mixed-effects Poisson models for secondary outcomes depending on whether the outcome is continuous, binary, or count data. A cost-effectiveness and cost-utility analysis will be undertaken.
\end{abstract}

Discussion: This definitive, national, multi-site trial is of sufficient size to evaluate MBT to inform policymakers, service commissioners, clinicians, and service users about its potential to treat offenders with ASPD and the likely impact on the population at risk.

Trial registration: ISRCTN 32309003. Registered on 8 April 2016.

Keywords: Antisocial personality disorder, Randomized controlled trial, Mentalization-based treatment, Probation, Personality disorder, Offenders, Aggression, Violence, Patient and public involvement, Economic evaluation

\section{Background}

Personality disorder is substantially overrepresented in offending populations. Multiple studies consistently report a high prevalence of personality disorder in offenders in general [1] and in individuals with convictions for violent offences in particular [2]. Antisocial personality disorder (ASPD) is the most common personality disorder in criminal justice settings [3, 4]. This disorder is characterized by a failure to conform to social norms with respect to lawful behaviours; irritability and aggressiveness; impulsiveness; disregard for the feelings and safety of others; and disregard for one's safety and for the consequences of one's behaviour $[5,6]$. The aim of this study is to establish if an intervention targeting the spectrum of symptoms and behaviours associated with ASPD is of value in improving individual mental health and reducing associated public health and social burden.

ASPD is associated with high levels of comorbid conditions [7-10]. Over 90\% of individuals with ASPD have at least one other psychiatric disorder [11], at least $50 \%$ have co-occurring anxiety disorders [12], and 25\% have a depressive disorder [13]. Men with ASPD are 3-5 times as likely to misuse alcohol and illicit drugs as those without ASPD [14], and women with ASPD have an even higher likelihood of drug and alcohol misuse [14, 15]. ASPD is also associated with physical disability [16] and premature mortality [7]. Men with ASPD have a higher rate of premature death than men of the same age without the disorder, due not only to an increased risk of suicide but also to reckless behaviours such as drug misuse and aggression [8].
The prevalence of ASPD has been reported as $0.6 \%$ in the UK general population [5], although it may be underdiagnosed in the community [17]. Nonetheless, there is a wide disparity between the prevalence of ASPD among the general population and among the offending population: in the UK prison population, just under two thirds of male remand prisoners, half of male sentenced prisoners, and one third of female prisoners meet diagnostic criteria for ASPD [4]. Although the prevalence of ASPD in probation services has not been fully explored, a screening study showed that nearly half of those on probation had probable personality disorder [18], an estimate likely to be higher among those supervised as 'high risk'. In addition, reforms to the UK probation system in 2013 mean that ASPD is likely to be even more prevalent in the high-risk population supervised under the $\mathrm{Na}$ tional Probation Service (NPS) in which the study is being conducted.

The contribution of ASPD to violent criminal behaviour is clear: it is associated with a significantly increased likelihood of committing violent crimes $[19,20]$ and is highly predictive of future violence, future reconviction or rapid re-incarceration upon release, and severity of recidivism [21, 22]. The social impact of individuals whose ASPD manifests in the form of violent criminal behaviour includes direct physical and emotional harm to victims, damage to property, use of police time, involvement with the criminal justice system, and increased use of healthcare facilities [23]. Finding an effective treatment to reduce aggression in individuals with ASPD has potential public health benefits, both directly, by improving the health of those with ASPD, and 
indirectly, through reducing the adverse consequences for victims, families, and communities.

Although the treatment of ASPD is a well-recognized priority, many studies evaluating interventions for antisocial behaviour have not looked at personality disorder diagnoses, and no intervention has been established as the treatment of choice for addressing the symptoms of ASPD. The paucity of studies in this area is notable [24]; only a small number of high-quality trials of specific therapies for ASPD have been conducted. We conducted a systematic literature search under National Collaborating Centre for Mental Health guidance, focusing on papers published from 2009 to 2016 (since the UK National Institute for Health and Clinical Excellence (NICE) review [5] was completed), using a strategy consisting of randomized controlled trial (RCT) and systematic review filters combined with subject heading and free-text phrases for ASPD. The search strategy was run using the standard mental health-related and allied health bibliographic databases (PsycINFO, EMBASE, AMED, and MEDLINEusing the Ovid interface). The search yielded 460 results after deduplication. After excluding results that were not journal papers (11), did not focus on ASPD (301), did not focus on quantitative treatment outcomes for existing ASPD (133), and did not differentiate ASPD from other personality disorders (10), only five results remained: three trials [25-27] and two Cochrane reviews [28, 29]. Of the trials, only one study was conducted in a community sample [25]; this was not a definitive RCT but an exploratory trial in a small sample of adult men with $\operatorname{ASPD}(N=52)$, which investigated the feasibility of carrying out a full RCT of cognitive-behavioural therapy versus treatment as usual. Four ongoing studies were also found by searching trial registries. These trials' sample sizes were relatively small: one of $N=114$, the others of $N<50$. Moreover, within their samples, these trials did not differentiate ASPD from other disorders such as borderline personality disorder (BPD) or paranoid personality disorder, or from autism. The search also revealed a number of metaanalyses, including a recent Cochrane review, which highlight the lack of evidence to support the use of any intervention for ASPD and urgently recommend that research be carried out to determine effective and cost-effective interventions [5, 28-31]. In this paper, we present the protocol for a large-scale RCT to generate new knowledge to address this gap in existing knowledge.

We propose the randomized evaluation of a psychotherapeutic model, mentalization-based treatment (MBT) for ASPD (MBT-ASPD). MBT is a manualized intervention that is based on the assumption that individual differences in the capacity to understand behaviour in terms of mental states arise as a result of variability in the social environment in early childhood, when mental state understanding of behaviour is normally acquired [32-34]. MBT integrates cognitive and relational components and provides a structured therapeutic process during which the patient's mind becomes the focus of treatment, centring on his/her capacity to accurately interpret the meaning of actions in terms of mental states (e.g. beliefs, thoughts, feelings, or desires). People with ASPD show impaired recognition of basic emotions [35], impaired capacity to link mental states to behaviour [36, 37], and difficulty with perspective-taking problems and in reading others' mental states [38-43]. They perform far worse than controls on subtle tests of mentalizing [44, 45]. These observations are consistent with a number of theories of antisocial behaviour $[46,47]$, including the deficit theory in the mentalizing literature, as well as the mentalizing model of antisocial behaviour, which is premised on the dysfunction of the attachment system that then temporarily inhibits affect regulation and mentalizing abilities [48-51]. Antisocial behaviour and violence tend to occur when an understanding of others' mental states is developmentally compromised (fragile) and prone to being lost when the attachment system is activated by perceived threats to self-esteem, such as interpersonal rejection or disrespect [52]. Normally, mentalizing (i.e. envisioning the subjective state of the victim) prevents interpersonal violence [53]; this means that individuals with vulnerable mentalizing capacities can be behaviourally volatile in moments of interpersonal stress. Supporting the capacity to identify others' emotions and intentions may not only assist social functioning but also reduce the risk of antisocial behaviour. Indeed, mentalizing has been shown to be a protective factor against aggression in people with violent traits [39]. Encouraging mentalizing has been shown to reduce school violence $[54,55]$, and MBT has demonstrated success in treating symptoms of impulsivity in individuals with comorbid BPD and ASPD [56]. Other studies of forensic patients with personality disorder have found that participants' views of the processes by which therapeutic changes occurred tended to identify realizations that in turn reflected improved mentalizing $[57,58]$.

Recognizing the therapeutic potential of MBT for ASPD, the developers have adapted MBT specifically for individuals with ASPD, in line with the NICE guideline recommendation that interventions for ASPD should be geared to enabling individuals to better examine their own states of mind and understand others' minds, and to behave more prosocially [5]. MBT-ASPD is a complex psychological intervention delivered using a combination of group and individual sessions [53, 59-61], which aim to enhance mentalizing by helping participants to develop metacognitive understanding of their difficulties with violence and achieve control over their aggression by addressing relevant drivers such as interpersonal 
misattributions (by improving interpersonal understanding through cognitive and affective identification with the experiences of others) and emotion dysregulation (by facilitating controlled and conscious mentalizing rather than automatic, non-reflective, non-conscious mentalizing).

\section{Aims}

The primary aim of the Mentalization for Offending Adult Males (MOAM) trial is to address the following research question: is probation as usual (PAU) supplemented with MBT more effective and cost-effective than standard PAU alone for reducing aggressive antisocial behaviour in male offenders under community supervision who meet DSM-IV diagnostic criteria for ASPD? As there is currently poor characterization of the primary and secondary care needs of people with ASPD, the trial will aim to investigate the impact of $\mathrm{MBT}$ on a range of health-related and behavioural outcomes, some of which are of interest irrespective of any diagnosis (e.g. quality of life) and some of which are particularly pertinent to ASPD and its symptoms (e.g. offending, violence, substance use, remission from categorically defined ASPD status), and to examine the mediators and moderators of these outcomes. The trial will also aim to establish the relative cost-effectiveness of MBT and PAU, taking into account costs incurred and the return on investment across health services, social services, the criminal justice sector, and voluntary sector services during the 24month period following randomization. Additionally, the trial will aim to analyse offender referral information to identify trends, calculate the size of the population likely to benefit from an intervention for ASPD, map outcomes from current interventions, and make recommendations about treatment groupings and targeting of services. Finally, the trial aims to generate and disseminate data that support policymakers, service commissioners, and service providers in making evidence-based decisions about planning and delivering services for offenders with ASPD on probation and in improving the management of mental health needs of offenders with ASPD in the community.

\section{Methods}

\section{Trial design}

The MOAM trial is a pragmatic, multi-site RCT comparing MBT with PAU for adult males meeting DSM-IV diagnostic criteria for ASPD [62], who are under community probation supervision. The design is a singleblind (with researchers being blinded) RCT of a 1-year programme of MBT-ASPD, which comprises weekly group sessions $(75 \mathrm{~min}$ ) and monthly individual sessions (50 $\mathrm{min}$ ), compared with the usual services provided by probation (i.e. PAU) for this client group. Typically,
PAU can include a variety of treatments, ranging from those that directly address personality disorder (e.g. schema-focused therapy) to those that address more specific criminological characteristics (e.g. anger management programmes, substance misuse interventions, domestic violence interventions), although in some services there are no targeted treatment options currently available for participants with a diagnosis of ASPD. This trial is unique among studies evaluating interventions for antisocial behaviour in that it is a full RCT with a large sample size, which targets ASPD as the primary diagnosis, and which differentiates ASPD from other mental health conditions.

\section{Ethics}

The study protocol was approved by the London South East Research Ethics Committee (reference number 14/LO/1696) and the National Offender Management Service (reference number 2014-315). Research and development approval has been sought and obtained for each trial site by the relevant NHS Trust and NPS lead in each geographical area. The trial sponsor, University College London, played no part in study design; collection, management, analysis, and interpretation of data; writing of the report; and the decision to submit the report for publication.

\section{Study setting}

This study involves two UK statutory services-the NPS in partnership with NHS Trusts-at 13 sites. The MBT clinical services, like other National Offender Personality Disorder community services, were designed to be delivered through existing community partnerships between Probation Trusts and health service providers. Sites in England and Wales were invited to bid for the clinical services in 2014, prior to the commencement of the RCT in 2016. The National Offender Personality Disorder Programme covers England and Wales, but not Scotland or Northern Ireland, hence the restriction of sites to England and Wales. Providers needed to already be part of the Offender Personality Disorder Pathway Strategy with an existing contract in place between NHS England and the Probation Trust to deliver the community service specification in addition to a subcontract with a health service provider. Services were also selected on the basis of (a) geographical spread across England and Wales, (b) demographic representativeness (urban vs rural), and (c) availability of participants for recruitment into the trial (favouring somewhat larger services). The 13 sites comprise the following: NPS London and the Tavistock and Portman NHS Foundation Trust in conjunction with Barnet, Enfield, and Haringey Mental Health NHS Trust; NPS London and the South London and Maudsley NHS Trust; NPS Lincolnshire and the Lincolnshire Partnership 
NHS Foundation Trust; NPS Merseyside and the Merseyside Care NHS Trust; NPS Devon \& Cornwall and the Devon Partnership NHS Trust; NPS London and the East London NHS Foundation Trust; NPS London and the Oxleas NHS Foundation Trust; NPS Lancashire and the Lancashire Care NHS Foundation Trust; NPS Staffordshire and West Midlands and the South Staffordshire and Shropshire Healthcare NHS Foundation Trust; NPS Nottinghamshire and the Nottingham Healthcare NHS Foundation Trust; NPS Gloucestershire and the Avon and Wiltshire Mental Health Partnership NHS Trust; NPS Wales and Hywel Dda University Health Board; and NPS West Yorkshire and Leeds \& York Partnership NHS Foundation Trust.

\section{Participants}

A total of 302 participants will be recruited, with approximately half of the consecutive qualifying cases being randomized to MBT and the other half to PAU. The unit of randomization is the individual participant.

Participants will be identified from a pool of offenders subject to statutory supervision by the NPS and assessed as having indications of personality disorder according to the Community Personality Disorder Pathways Service Specification of the Offender Personality Disorder Pathway Strategy following case identification, consultation, and formulation.

\section{Eligibility criteria}

Inclusion and exclusion criteria have been carefully selected on the basis of previous research and clinical experience to provide an appropriate balance between homogeneity (to ensure that the treatment under investigation is appropriately aimed toward the diagnostic needs profiles of those within the trial) and heterogeneity (to accurately reflect the mix of antisocial offenders under the management of community probation and to ensure that the results of the trial are generalizable to the wider population of people diagnosed with ASPD) within the sample, as well as to enable comparability with other trials investigating the treatment of aggression and of antisocial behaviour [25, 63-65].

All participants will meet the following inclusion criteria:

- Male and aged 21 years or over

- Subject to statutory supervision by the NPS

- At least 6 months remaining of their licence or community sentence

- Fulfilling DSM-IV diagnostic criteria for ASPD (assessed by the Structured Clinical Interview for DSM-IV (SCID) Axis II Disorders (SCID-II)) [66]

- Score of at least 15 on the Overt Aggression Scale Modified (OAS-M) [67]
- Adequate English language and cognitive capacities to participate in informed consent and group therapy.

The exclusion criteria applied will be minimal and are as follows:

- Serving a conviction for child sexual offences

- Primary diagnosis of a psychotic disorder or neurodevelopmental disorder.

\section{Sample size and power calculation}

The sample size $(N=302)$ has been chosen to have $90 \%$ power, at a two-sided 5\% significance level, to detect a significant difference between groups when the change in OAS-M score is on average 10 points less in the MBT arm (with a standard deviation of $20)$ at the primary endpoint 24 months after randomization. Hollander et al. [68] reported the pooled standard deviation of the change in OAS-M score from baseline to 10-week follow-up as being just over 9 for the pharmaceutical intervention assessed in that study. Due to the longer follow-up period of the present trial and the nonpharmaceutical nature of the planned intervention, we have chosen to increase the standard deviation used in our power calculation to 20 . To take into account potential clustering by clinician in the MBT arm, we assume the intraclass correlation coefficient to be 0.05. In this case, using established methods to take account of clustering [69], 95 participants per arm will give $90 \%$ power. We anticipate attrition and dropout to be $37 \%$, based on several sources including our own preliminary work and several larger data sources (a meta-analysis of attrition rate in offender treatment literature [70], a systematic review of non-completion of treatment for personality disorder [71], and an empirical evaluation of treatment disengagement in offenders with personality disorder [72]). To account for this loss, initial recruitment of 151 participants per arm is anticipated to result in 95 participants remaining per arm after attrition and dropout. Our target sample size is therefore 302 participants, which will give sufficient power to detect a medium effect size of 0.5 change in participants' OAS-M score.

Demographic and clinical details from those refusing randomization will be retained to explore whether the trial population is representative. To assess treatment acceptability, we will compute the proportion of referred individuals who never attended, the number of sessions attended, and the number who drop out of treatment or request to be referred to other treatments. All participants who do not formally withdraw from the study will be followed up on routine measures of outcome. 


\section{Peer researchers}

A crucial aspect of this trial design is the use of peer researchers. This approach enables individuals with similar key criteria to the participant group to play an active role in the research process, by taking on the role of researchers. There are a number of potential benefits associated with using peer researchers, including the increased likelihood of accessing people and topics that traditional research and service staff may not be able to reach [73] and the potential to enhance the accuracy and validity of participant data through peer researchers being better placed to put participants at ease, thus stimulating free discussion and more open dialogue [74]. Peer researchers may also reduce the power differentials between researchers and participants, thereby facilitating trust and rapport and promoting honest disclosure of sensitive information [75]; this is particularly important in our target population, given that those involved in the criminal justice system often have an entrenched distrust of authority. In this study, peer researchers with lived experience of the criminal justice system, provided by the charity User Voice, will work alongside traditional research assistants under robust supervision arrangements to conduct baseline and outcome assessments. Peer researchers will be expected to have successfully reintegrated into society and have prior experience of an engagement role.

\section{Recruitment and baseline procedures}

Figure 1 shows the expected flow of participants from recruitment through to the end of the study, based on observed screening and referral data to date. In addition to aspects that apply to recruitment for any trial (e.g. the clear application of eligibility criteria, a standard procedure for obtaining informed consent), recruitment for this trial will be especially sensitive to the community probation context and will be based on effective partnerships with referral agencies and strong relationships with the participants. Consequently, the trial team has developed strong collaborative relationships with the MBT team at each site to achieve the high levels of accrual necessary to ensure sample comparability and reasonable generalizability.

We have developed a multiple gating procedure for recruitment. Decisions about eligibility for the trial will be made at three key points: (1) by the referring agencies (i.e. individual Offender Managers across each site) following a discussion with the Specialist Offender Manager (SOM) in the MBT team to identify ostensibly suitable potential participants; (2) through an explanatory meeting between the offender, the MBT team, and the research team following the initial referral, to explain the research trial, invite participation, and answer any questions about the clinical services potentially available to the participant; (3) as a result of discussions between the MBT clinicians, Assistant Psychologist, and Research Manager following an in-depth clinical assessment and formal diagnostic evaluation, to screen out offenders who do not meet the eligibility criteria.

Experience suggests that each of these screens tends to reveal different criteria for ineligibility, and their use in combination minimizes the (considerable) effort of recruitment. For example, the SOM tends to identify and screen out cases whose offending history includes child sex offences, whereas discussions between the MBT and research teams and the potential participant most commonly identify and screen out individuals who are insufficiently motivated to engage with group treatment. The clinical assessment reveals offenders who are ineligible due to their lack of suitability for tolerating a group setting or psychotherapeutic treatment in general, and the formal diagnostic evaluation is necessary to confirm the individual's psychiatric diagnosis.

\section{Screen 1}

The SOMs in the MBT teams will work across each of the 13 sites, taking referrals from Offender Managers who will put forward individuals they deem agreeable to being approached about participation and who have adequate English and cognitive capacities to participate in informed consent and group therapy. SOMs will be available to discuss cases that potentially meet the eligibility criteria for MBT. In addition, the SOM and Assistant Psychologist at each site can directly screen for suitable cases by reviewing their caseload against the inclusion and exclusion criteria, before approaching the respective Offender Manager to make a referral.

The decisions made by the SOM constitute the first screen. A standard referral form for each locality is used in liaison with the research team, including specific information pertaining to inclusion and exclusion criteria.

\section{Screen 2}

Following acceptance of the referral by the MBT team, a meeting will be arranged between the MBT and research teams and the offender, to inform the offender about the trial and the opportunity to participate. The discussions at this meeting include what the MBT intervention would entail and the alternative PAU path (the best available alternative treatment at that locality; see below) should the offender not be randomized to receive MBT. Offenders will be provided with participant information sheets that have been co-designed with input from exoffenders to make them as useful and accessible as possible. Offenders will be given a minimum of $24 \mathrm{~h}$ to consider participation. 


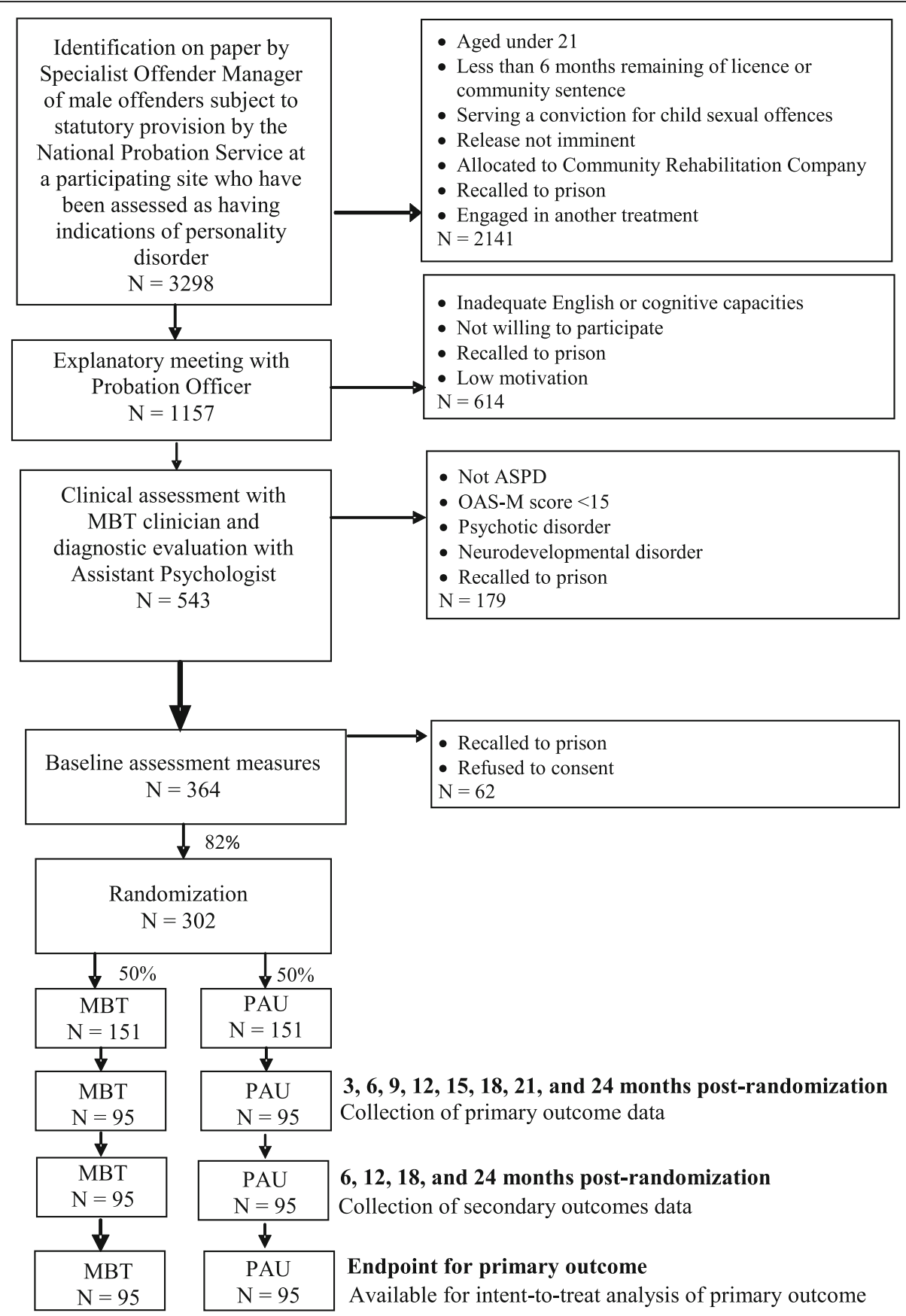

Fig. 1 Expected flow of participants from recruitment through to the end of the study

\section{Screen 3}

If the offender decides to participate following the explanatory meeting, an in-depth clinical assessment and formal diagnostic evaluation will be arranged. MBT clinicians will conduct history-taking, administering the SCID-II psychosis screen, and ascertaining the offender's ability to engage in psychotherapeutic treatment and tolerate a group setting.
Consent forms will be signed by both the offender and the site's lead MBT clinician, who will assess the offender's capacity to provide informed consent. Consenting to the trial includes providing permission to access police records, remaining in effect for 2 years, and health records, remaining in effect for 5 years.

The site's Assistant Psychologist will administer the SCID-II screen and the OAS-M. If all of the inclusion 
criteria and none of the exclusion criteria are met, the participant will progress to the next stage of the research process to complete the baseline measures.

\section{Baseline measures}

For offenders who sign the consent forms, the researcher will administer pre-randomization questionnaires and measures to be completed during this contact (i.e. before group assignment). When all the instruments have been completed and eligibility for the trial has been confirmed, randomization will be performed and details communicated to the referrer, MBT team, and participant within $48 \mathrm{~h}$.

\section{Randomization and procedures to minimize bias}

Following consent and baseline measures, a trial identification number will be assigned to eligible consenting participants and a recruitment 'dam' created behind which a participant pool will be built up, from which participants will be randomized to enable MBT groups to start. Once groups have started, sequential assignment will allocate participants to MBT or PAU in a 1:1 ratio. Randomization will be undertaken off-site, using a dynamic adaptive allocation algorithm [76] accessed by a secure web portal to the system held at NWORTH Clinical Trials Unit (CTU) and maintained by a statistician independent of the analysis and research teams to ensure blinding. Stratification will be by site, age (21-25; 26-39; 40+ years), probation order type (Community Sentence; On Licence Post-Prison), and time remaining on probation (less than 12 months; 12 months and over). Treatment site is included in the minimization stratification to control for differences between sites. To minimize bias that could arise from knowledge of treatment allocation, researchers will be blind to participants' treatment. The allocation to treatment arms will not be modified unless the local principal investigator, with the agreement of the clinical supervisor or the Trial Steering Committee (TSC), identifies severe risks of adverse events (homicide, suicide, serious injury, etc.) associated with the experimental treatment. Experimental treatment will be discontinued if the participant breaks the conditions of probation and is asked to serve the remainder of their prison sentence for a time which prevents any further attendance or if the participant commits further offences and receives a custodial sentence for the fresh conviction that is longer than the remaining time of treatment.

\section{Planned interventions}

Implementing standard PAU or PAU supplemented with MBT will not require alteration to usual pathways (including use of any medication), and these will continue for both trial arms.
PAU

Participants randomized to receive PAU will remain under the supervision of the NPS for the duration of their licence or community sentence. PAU is a wellspecified and stringently monitored standard procedure used in the management of offenders under community supervision. It consists of regular contact with a designated Offender Manager for the duration of the offender's licence or sentence, and includes referral for additional interventions as indicated in Ministry of Justice guidance. PAU participants will be free to be referred by their Offender Manager for any locally available treatment (except MBT) designed to reduce reoffending (e.g. anger management programmes, substance misuse interventions). Provision of these interventions is not uniform across the UK but dependent on local availability; in some areas, there may be no targeted interventions available. Unlike MBT, none of the interventions that participants may receive under PAU involve targeting the capacity to envision mental states more accurately. Details of services accessed under PAU will be documented by the SOM to facilitate economic data collection and evaluation. Additional site-specific strategies (e.g. separate supervision groups) will ensure that MBT principles and practice do not directly influence the management of participants randomized to PAU. PAU will last for 12 months, after which participants who still have time remaining on their licence or community sentence will remain under the supervision of the NPS for the duration. Participants whose licence expires during the trial period may remain under the NPS on a voluntary basis to complete the 12-month period.

\section{PAU supplemented with MBT-ASPD}

The treatment intervention is a 1-year programme of group and individual sessions of MBT-ASPD. Participants randomized to MBT-ASPD receive weekly group therapy for $75 \mathrm{~min}$ and monthly individual therapy for 50 min. Each MBT-ASPD group is run by two trained MBT clinicians and contains a maximum of eight participants, using an 'open' rolling entry group, with new participants joining as others leave. The group process enables participants to challenge each other about their understanding of current self-identified critical interpersonal experiences and focuses particularly on elaborating the mental state background of interactions characterized by conflict that might normally trigger aggression. The content of the group therapy sessions is steered by the clinicians toward encouraging the participants to talk about their mental states related to recent violent incidents by linking their actions to a broader range of their current subjective and emotional experience (i.e. to 'mentalize' them). A monthly 50-min individual session provides an opportunity for participants to address 
personal issues that have occurred in the group. Many participants find it more difficult to talk in front of other members of the group about the relationship difficulties they experience in their external lives, and may use the individual sessions to do this too. Both group and individual sessions focus on identifying thoughts and feelings associated with aggressive impulses, with particular emphasis on understanding emotional cues, recognizing emotions in oneself and others, understanding others' experiences in relation to one's own, and clarifying threats of loss to mentalizing in the context of life experiences, both currently and historically.

All participants randomized to this arm of the study have an allocated clinician. Staff providing MBT sessions are chartered health professionals with a core clinical training background (e.g. clinical psychology, nursing, medicine) who have undergone an MBT training course. Where possible, recently trained clinicians are paired with an experienced clinician to provide group MBT.

The evaluation of the adequacy of protocol delivery and adherence to the model will include treatment integrity measures based on the MBT Adherence and Competence Scale [77], applied to video recordings of a random sample of sessions. This instrument enables coders to rate therapist activity and appropriateness in five key domains of MBT with ratings on Likert scales supported with anchored descriptors. As clinicians will be specially trained for the trial, it is critical to separately evaluate both clinician adherence and level of clinician competence, which will be based on the independent rating of randomly selected session recordings. The scale measures both the frequency of therapists' actions (adherence) and the quality of the delivery of those actions (competence). Adherence refers to the number of items used within a domain and their frequency of use; quality refers to the clinician's demonstration of how the interventions are delivered in terms of (a) expertise, competence, and commitment; (b) timing; (c) taking account of the context and content of the session; (d) matching the mentalizing state of the patient; (e) responding to where the patient appears to be; and (f) an extensiveness component in terms of items used when working in the domain. A definition of skill/quality is given for each domain.

Clinicians with average scores above 3.5 will be considered to have achieved acceptable levels of adherence and competence. Adherence will be monitored on a monthly basis through clinical supervision sessions using the MBT Adherence and Competence Scale, based on independent rating of randomly selected session video recordings, to ensure that clinical staff are consistently on-model.

\section{Assessments and outcome measures}

To maximize the validity of the outcome evaluations, assessments will be made across multiple domains using multiple methods and sources.

\section{Primary outcome}

The primary outcome is the frequency of aggressive antisocial behaviour as measured by an adapted version of the OAS-M and is collected every 3 months. The OAS-M provides information to establish frequencies of verbal aggression and physical aggression against objects, against others, and against the self. The OAS-M is a robust semi-structured interview and is the most widely used measure of aggression in trials and clinical studies in this area. It has been used with many different outpatient populations, including those with Cluster B personality disorders. It is one of the only measures of aggression that assesses actual aggressive behaviour, and its format offers several advantages over most available instruments for assessing anger and aggression, including allowing the probing of vague and inconsistent answers [67]. It is therefore well suited to accurate evaluation of aggressive behaviour and of change over time, both as a research instrument and as a complement to many of the current self-report measures. In agreement with the developer of the measure, the OAS$\mathrm{M}$, adapted for the current study, uses a weighted scale taking the number of reported endorsed items and multiplies them in accordance with the severity of each item. Aggression is the primary outcome in this study as it is the key characteristic of ASPD, the primary driver of interpersonal violence, and a key contributor to health status $[78,79]$. The primary endpoint is at 24 months post-randomization. As a secondary outcome, the adapted OAS-M is collected at other time points $(3,6,9$, 15 , and 18 months).

\section{Secondary outcomes}

The domains that the investigators consider key to the intervention are violence, anger, offending behaviour, impulsivity, alcohol and substance misuse, self-harm and suicidality, general health and wellbeing, service satisfaction and engagement outcomes, and remission in ASPD diagnostic status. These secondary outcomes will be assessed by using objective and self-report measures. Estimates of the population at risk resource use data relevant to conducting a comprehensive economic evaluation will be collected alongside these outcomes, as well as data on variables associated with putative mechanisms of change.

\section{Objective measures}

Objective outcomes of health service use will be collected from record linkage using participants' NHS 
numbers at 6-monthly intervals during the intervention and the follow-up period until the 24-month follow-up point.

Objective outcomes of offending behaviour, including withdrawal of licence, arrests, and reconvictions, will be collected from police computer records at 6-monthly intervals, for the 6 months before randomization and during the intervention and follow-up period until the 24month follow-up point. The number of records of offending behaviour (count data) will be obtained, and 6-month periods free of any offending behaviour will also be recorded (binary data). Data will be obtained from participants' records on nDelius (the national public sector offender case-management system) and the Police National Computer database; these records detail information on breaches of licence conditions, police contacts, charges, court appearances, criminal orders, arrest rates, and frequency, severity, and type (violent/nonviolent) of offending behaviour.

These objective outcomes of health service use and offending behaviour will be available regardless of loss to follow-up; this will allow the use of multiple imputation techniques. If loss to follow-up is considerably different between arms and the primary outcome is significant, we will perform a sensitivity analysis to the impact of missing data not being missing at random, as described by White et al. [80].

\section{Self-report measures}

Researchers will administer pre-testing questionnaires during the initial contact with participants after they have given consent to participate in the trial, before group assignment (as described above). Follow-up assessments will be made at 3-month intervals for the primary outcome measure and for secondary outcome measures assessing violence, intimate partner violence, and anger; at 12-month intervals for the secondary outcome measures assessing categorically defined ASPD and rates of desistance; and at 6-month intervals for all other secondary outcome measures. Assessments will continue for the duration of the 12-month treatment and for 12 months post-treatment. Self-report measures of violence, offending behaviour, substance misuse, selfharm, and suicidality, as well as general health and wellbeing, service satisfaction, and engagement, will be collected.

Violence will be measured by the MacArthur Community Violence Screening Instrument [25, 81]. This measure assesses the presence, severity, and frequency of violent behaviours and is one of the few instruments available to differentiate between general aggression and specific interpersonal violence [82].

Intimate partner violence will be measured by the Short Form of the Revised Conflict Tactics Scales. This measure includes scales to measure physical assault, psychological aggression, and sexual coercion against a partner in a dating or marital relationship.

Anger will be measured by the State-Trait Anger Expression Inventory-2 (STAXI-2) [83, 84]. The STAXI-2 is a psychometrically robust questionnaire [85] designed to assess the experience, expression, and control of anger, which commonly drives aggression.

Alcohol use, which has been strongly associated with aggression and offending [86], will be measured by the Alcohol Use Disorders Identification Test (AUDIT) [85, 87]. The AUDIT is widely used as an alcohol use screening measure. It assesses three aspects of drinking: quantity and frequency, indicators of dependence, and adverse consequences suggesting harmful use.

Drug use, which is similarly linked to aggression and offending [88], will be measured by the Drug Use Disorders Identification Test (DUDIT) [89], which assesses frequency of use, indicators of dependence, and adverse consequences suggesting harmful use. The DUDIT has several advantages over other instruments, including a brief administration time (5 min) and its focus on recent use and consequences.

Self-harming behaviours, an indicator of impulsivity and a target of MBT, will be measured by the Self-Harm Inventory [90], a widely used measure that yields a severity of self-harm score based on the frequency of a wide range of impulsive behaviours.

Suicidal behaviour, a further complication of offending behaviour with ASPD [91], will be measured by the Suicidal Behaviours Questionnaire-Revised [92]. This instrument assesses four different dimensions of suicidality, including frequency of suicidal ideation and threat of suicide attempt.

Health-related quality of life will be measured by the EQ-5D-5L [93, 94]. The EQ-5D-5L is a standardized instrument for use as a measure of health outcome that is applicable to a wide range of health conditions and treatments. In this study, it will be used to provide a generic measure of health for both clinical and economic appraisal.

Mental health status will be measured by the Symptom Checklist-90 - Revised (SCL-90-R) [95]. The SCL90-R assesses psychological distress in terms of nine primary symptom dimensions, including depression, anxiety, hostility, and paranoid ideation-all potential drivers of aggression.

Personality dysfunction will be measured by the Personality Inventory for DSM-5 - Brief Form (PID-5-BF) [96]. This 25-item personality trait assessment scale assesses negative affect, detachment, antagonism, disinhibition, and psychoticism and will provide a means of assessing change in the trait domains that are most prominent in ASPD. 
Categorically defined ASPD will be measured by the ASPD module of the SCID-II, to assess whether MBT produces remission in ASPD diagnostic status.

Mentalizing capacity will be evaluated by the 16-item Brief Reflective Functioning Questionnaire [97] to assess whether MBT's proposed mechanism of change is in line with any symptomatic improvements.

Rates of desistance will be measured by the Redemption and Condemnation Self-Narrative Scale version 2 to assess whether MBT increases rates of desistance in the participants.

\section{Clinician-rated measures}

Clinicians (therapists, Assistant Psychologists, and Offender Managers) will use the Service Engagement Scale [98] to determine the level of engagement of participants, as rated on four subscales: availability, collaboration, help-seeking, and treatment adherence.

\section{Economic evaluation}

Health economic analysis will be conducted by King's Health Economics at the Institute of Psychiatry, Psychology and Neuroscience, King's College London, and will explore the relative costs and cost-effectiveness of $\mathrm{MBT}+\mathrm{PAU}$ versus PAU. Although there is a preference for economic evaluations carried out on NHS services to take an NHS and personal and social services cost perspective, as recommended by NICE [99], in this case our primary analysis will take a wider societal perspective, including all healthcare, social and personal services, voluntary sector services, costs to the criminal justice sector, including probation, and the costs resulting from any crimes committed. The wider perspective is relevant and valid here because the interventions being compared are delivered in a criminal justice setting and the hypothesized impact will fall across both healthcare and criminal justice. A secondary analysis will consider the NHS/personal and social services costs only. Data on MBT contacts will be collected directly from sites, to avoid participants revealing their group allocation to the researchers. Data on the use of all other services, including PAU, will be collected in interviews using the Secure Facilities Service Use Schedule [100]. This instrument has been adapted to the current study population to ensure comprehensive coverage and face validity. Information on criminal activity will be extracted from police records. The cost of the trial interventions will be calculated through a detailed micro-costing (or bottom-up) approach using standard costing methodology [101], which will involve estimation of the indirect time spent on individual cases, including preparation, meetings, telephone calls, and supervision, as well as detailed recording of direct face-to-face contact. Other unit costs will be sourced from routine sources for the unit cost year 2017-2018 [102-104].

\section{Follow-up assessment}

Follow-up assessments will be conducted at 3-month intervals for 24 months post-randomization. Primary outcome measures will be collected every 3 months and secondary outcome measures every 6 months. Table 1 shows a detailed outline of the planned measures at each follow-up point throughout the trial.

\section{Data management \\ Collection}

Outcome measures data will be recorded using the $\mathrm{Pa}$ tient Owned Database (POD), an internet-based highsecurity data collection and management software. The use of POD automatically scores and transmits the data to the data-analytic site without human interference reducing the risk of data entry error. Once entered, the data will be exported and cleaned by the CTU ready for analysis by the trial statistician. In line with a data sharing agreement with the NPS and UCL Data Protection Policy, participants' personal data will be collected and securely stored in password-protected files only accessible by members of the research team during the course of the study. At the end of the trial, all data will be archived in a safe and secure off-site location.

\section{Auditing}

The quality of data will be routinely monitored by the Research Manager throughout the duration of the trial. In addition, data quality will be monitored quarterly by an independent audit group, which will be responsible for reviewing data collection and completeness rates.

\section{Statistical analysis}

All planned statistical analyses will be specified in a Statistical Analysis Plan that is agreed prior to unblinded information being made available to the trial statistician. Primary analyses will be on an intent-to-treat basis, with per-protocol (all participants who were randomized, who met inclusion/exclusion criteria, and who attended at least $35 \%$ of planned meetings with their parole officer) analyses undertaken as secondary. The characteristics of the treatment groups will be described at baseline. Preliminary analysis will investigate the pattern of missing follow-up data.

\section{Primary outcome}

The primary outcome (OAS-M) at the various follow-up times will be analysed using a hierarchical mixed-effects linear regression with baseline OAS-M, length of sentence for index offence, and the stratification factors used in the randomization (except for site) as fixed effects. Patient ID 
Table 1 Schedule of assessment administration for a randomized controlled trial of mentalization-based treatment for antisocial personality disorder

\begin{tabular}{|c|c|c|c|c|c|c|c|c|c|c|c|}
\hline & \multicolumn{11}{|c|}{ Study period } \\
\hline & \multirow[t]{2}{*}{ Enrolment } & \multirow[t]{2}{*}{ Allocation } & \multicolumn{9}{|c|}{ Post-allocation } \\
\hline & & & $\overline{\mathrm{T} 1}$ & $\mathrm{~T} 2$ & T3 & T4 & T5 & T6 & T7 & T8 & T9 \\
\hline \multicolumn{12}{|l|}{ ENROLMENT } \\
\hline Eligibility screen & $x$ & & & & & & & & & & \\
\hline Informed consent & $x$ & & & & & & & & & & \\
\hline Allocation & & $x$ & & & & & & & & & \\
\hline \multicolumn{12}{|l|}{ INTERVENTION } \\
\hline MBT & & & $x$ & $x$ & $x$ & $x$ & $x$ & & & & \\
\hline PAU & & & $x$ & $x$ & $x$ & $x$ & $x$ & & & & \\
\hline \multicolumn{12}{|l|}{ ASSESSMENTS } \\
\hline \multicolumn{12}{|l|}{ Questionnaires-participant } \\
\hline Overt Aggression Scale - Modified & & & $x$ & $x$ & $x$ & $x$ & $x$ & $x$ & $x$ & $x$ & $x$ \\
\hline EQ-5D-5L & & & $x$ & & $x$ & & $x$ & & $x$ & & $x$ \\
\hline Symptom Checklist-90-Revised Short Form & & & $x$ & & $x$ & & $x$ & & $x$ & & $x$ \\
\hline Alcohol Use Disorders Identification Test & & & $x$ & & $x$ & & $x$ & & $x$ & & $x$ \\
\hline Drug Use Disorders Identification Test & & & $x$ & & $x$ & & $x$ & & $x$ & & $x$ \\
\hline State-Trait Anger Expression Inventory & & & $x$ & $x$ & $x$ & $x$ & $x$ & $x$ & $x$ & $x$ & $x$ \\
\hline MacArthur Community Violence Screening Instrument & & & $x$ & $x$ & $x$ & $x$ & $x$ & $x$ & $x$ & $x$ & $x$ \\
\hline Short Form of the Revised Conflict Tactics Scales & & & $x$ & $x$ & $x$ & $x$ & $x$ & $x$ & $x$ & $x$ & $x$ \\
\hline Self-Harm Inventory & & & $x$ & & $x$ & & $x$ & & $x$ & & $x$ \\
\hline Suicidal Behaviours Questionnaire - Revised & & & $x$ & & $x$ & & $x$ & & $x$ & & $x$ \\
\hline Secure Facilities Service Use Schedule & & & $x$ & & $x$ & & $x$ & & $x$ & & $x$ \\
\hline Personality Inventory for DSM-5-Brief Form & & & $x$ & & $x$ & & $x$ & & $x$ & & $x$ \\
\hline SCID-II, ASPD Module & & & $x$ & & & & $x$ & & & & $x$ \\
\hline Brief Reflective Functioning Questionnaire & & & $x$ & & $x$ & & $x$ & & $x$ & & $x$ \\
\hline Redemption and Condemnation Self-Narrative Scale version 2 & & & & & & & $x$ & & & & $x$ \\
\hline \multicolumn{12}{|l|}{ Questionnaires-clinician } \\
\hline Service Engagement Scale & & & & & $x$ & & $x$ & & $x$ & & $x$ \\
\hline National Probation Resources, Evaluation and System Schedule & & & $x$ & & $x$ & & & & $x$ & & $x$ \\
\hline \multicolumn{12}{|l|}{ Police and health data } \\
\hline Police records & & & $x$ & & $x$ & & $x$ & & $x$ & & $x$ \\
\hline Health records & & & $x$ & & $x$ & & $x$ & & $x$ & & $x$ \\
\hline
\end{tabular}

and site will be included as random effects. A treatment effect parameter for each follow-up time will be included, although the primary one of interest will be treatment effect at 24 months. Two-sided $p$ values less than 0.05 will be classed as significant.

\section{Secondary outcomes}

For the secondary outcomes, we will use mixed-effects linear regression for continuous outcomes, mixed-effects logistic regression models for binary outcomes, and mixed-effects Poisson models for count data such as number of offences. Missing outcome data due to dropout will be accounted for by the mixed-effects model under a missing-at-random assumption; missing covariates will be accounted for using multiple imputation.

\section{Moderators of outcomes}

The trial will evaluate the following as potential moderators for the primary and any secondary outcomes that are significantly different between arms: age, type of probation, length of probation, anxiety measured by the SCL-90-R, Axis II comorbidities measured by the SCID, $\mathrm{p}$ factor scores derived from a bi-factor analysis of the 
self-report outcome measures, baseline alcohol use measured by the AUDIT, and baseline drug use measured by the DUDIT.

Moderators will be tested by including interaction parameters in the model and testing them for significance.

\section{Mediators of outcomes}

We will test the following as mediators (if there is a significant difference between arms in the mediating variable): alcohol use (AUDIT score); drug use (DUDIT score); mood (scores on the anxiety and depression subscales of the SCL-90-R); mentalizing (Brief Reflective Functioning Questionnaire score).

We will also test the proportion of MBT planned sessions attended as a post-baseline effect modifier (as it is only collected in the MBT arm).

\section{Economic evaluation}

Mean total costs for the two groups at 24-month followup will be compared using a regression model with baseline costs as a covariate, an approach used despite the likely skewed distribution of the data because of the importance of the arithmetic mean in using the results for policymaking [105]. To test the robustness of the normality assumption, bootstrapped confidence intervals for the regression model will be also be calculated [106]. Quality-adjusted life years (QALYs) will be calculated from EQ-5D-5L health status using UK values for utility status [107] and assuming a linear change in utility over time. A cost-utility analysis will combine costs with the QALYs, a cost-effectiveness analysis will combine costs with aggressive behaviour and incremental costeffectiveness ratios, and cost-effectiveness acceptability curves will be generated. Sensitivity analyses will include a test of the impact of the intervention costs on the cost-effectiveness results, and the impact of missing data, tested using multiple imputation of missing cost items.

\section{Oversight and monitoring Oversight committees}

The study will be overseen by two oversight committees: the TSC and a Data Monitoring and Ethics Committee (DMEC). The TSC will be chaired by an independent expert and will be responsible for monitoring progress of the study and reviewing amendments to the trial protocol. Committee members will include experienced clinicians, commissioners of forensic mental health services, criminal justice professionals, and service user representatives. A group of clinicians with relevant experience in the area and expert trial statisticians will form the independent DMEC, which will be responsible for monitoring recruitment and follow-up rates across both arms of the trial, as well as serious adverse events (SAEs) and ethical concerns. Minutes from the DMEC will be shared with the TSC chair following each meeting, and the TSC will report directly to the funder.

\section{Adverse events}

For this study, adverse events will be defined as immediate risk of harm to the participant or another person and will be collected after the participant has provided consent and enrolled in the study. Each event will be logged by the research team following contact with the participant and regularly reviewed by the Research Manager, Clinical Lead, and Chief Investigator. If an adverse event meets the criteria for a SAE between study enrolment and the 24-month data collection time point, the event will be logged and reported to the DMEC for review. SAEs occurring after a participant is discontinued from the study will not be reported unless the investigators feel that the event may have been caused by a study protocol procedure.

\section{Dissemination policy}

The results of the study will be disseminated through publications in peer-reviewed journals as well as presentations, newsletters, and articles for the probation and prison system and third sector organizations working within the criminal justice system.

\section{Discussion}

This study will address key gaps in the offending and health literature. To date, there have been very few highquality trials with a sufficiently powered sample size that have evaluated therapeutic interventions for offenders with a primary diagnosis of ASPD. The MOAM trial is the first large-scale trial of treatment for offenders with ASPD in the community and has a number of strengths compared with other trials of interventions for antisocial behaviour. First, it is a definitive RCT, conducted in real-life community settings, at multiple sites with a representative geographical spread and a range of clinicians from diverse disciplinary backgrounds. Second, the sample size is sufficient to yield $90 \%$ power, even after accounting for a potentially high attrition rate. Third, its sample is selected on the primary diagnosis of ASPD as fully differentiated from other mental health conditions. Fourth, the trial design incorporates the innovative use of peer researchers as a strategy to enhance the accuracy and validity of participant data. Finally, the measures employed will enable us to look beyond recidivism and will capture the effects of MBT on a wide range of secondary health and behavioural outcomes, including diagnostic change of ASPD itself.

Aggressive antisocial behaviour has been chosen as the primary outcome for this trial, in light of it being a core feature of ASPD, an indicator of emotional wellbeing, and a frequent cause of grounds for arrest. A range of 
secondary outcomes have also been chosen in order to evaluate MBT's possible impact on the wide range of symptomatology and health outcomes experienced by individuals with ASPD, including impulsivity, offending behaviour, alcohol and substance misuse, self-harm and suicidality, and general health and wellbeing. The MOAM trial will also provide accurate information about the services accessed under PAU. A comprehensive cost-effectiveness evaluation will be undertaken to examine both costs offset and costs saved in relation to having received MBT or PAU. Thus, the trial will provide unprecedented data not only on the clinical effectiveness and cost-effectiveness of MBT-ASPD, but also on the clinical effectiveness and cost-effectiveness of the usual services available to this group while on probation. Furthermore, the trial will analyse offender referral information to identify trends, calculate the size of the population likely to benefit from an intervention for ASPD, and make recommendations about treatment groupings and targeting of services.

Information to date suggests that the trial protocol detailed above is acceptable to all the sites and that recruitment, data collection, and training are feasible in all locations. Preliminary data on participant engagement indicate that, of participants randomized to MBT, an average of 55-65\% attend group MBT sessions. Preliminary data indicate that one third of participants drop out at some point following randomization, entirely consistent with attrition rates reported in other studies involving treatment for offenders and patients with personality disorders [70-72]. For participants who drop out, the average length of engagement with the trial is 3-6 months; the most common reasons for dropout thus far are lack of motivation (58\%), recall to prison (35\%) and clinicians' discretion due to repeated nonattendance of MBT sessions (7\%).

The authors acknowledge that there is such a scarcity of evidence for effective interventions for ASPD that additional trials are greatly needed. Beyond the completion of the MOAM trial, the authors recommend further high-quality RCTs incorporating the following adaptations: minimal involvement of the intervention developers, to minimize bias; a female sample, to improve generalizability of results; longer-term follow-up, to determine whether differential effects are maintained; and investigation of the impact of differences in clinicians' training, experience, and supervision, to determine minimal training and supervisory standards and competences for effective delivery of treatment.

\section{Trial status}

The trial is in its fifth year. Sites were launched in four phases between January and September 2016, and recruitment ended in August 2018. Treatment and follow-up data collection are currently in progress. The protocol paper was submitted after the end of recruitment as the primary outcome, frequency of aggressive antisocial behaviour, is measured at the last follow-up meeting 24 months post-randomization. Outcome data will be available 24 months post-randomization of the last participant.

Trial protocol version: v6.0, 2 December 2018.

\section{Abbreviations}

ASPD: Antisocial personality disorder; AUDIT: Alcohol Use Disorders Identification Test; BPD: Borderline personality disorder; CTU: Clinical Trials Unit; DMEC: Data Monitoring and Ethics Committee; DUDIT: Drug Use Disorders Identification Test; MBT: Mentalization-based treatment; MBTASPD: Mentalization-based treatment for antisocial personality disorder; NICE: National Institute for Health and Clinical Excellence; NPS: National Probation Service; OAS-M: Overt Aggression Scale - Modified; PAU: Probation as usual; PID-5-BF: Personality Inventory for DSM-5-Brief Form; QALY: Qualityadjusted life year; POD: Patient Owned Database; RCT: Randomized controlled trial; SAE: Serious adverse event; SCID: Structured Clinical Interview for DSM-IV; SCL-90-R: Symptom Checklist-90 - Revised; SOM: Specialist Offender Manager; STAXI-2: State-Trait Anger Expression Inventory-2; TSC: Trial Steering Committee

\section{Acknowledgements}

The authors wish to thank User Voice for their support in collaborating on this trial, their contribution to peer-led research, and their unwavering commitment to optimal service user involvement; Zoe Hoare at NWORTH Clinical Trials Unit for providing valued methodological advice and for active practical support regarding participant randomization, quality assurance, data extraction, and management; Sarah Skett and Nick Joseph for their support with implementing a randomized controlled trial in the criminal justice system; and London Probation Pathways and Penrose for helping us identify service user experts for consultation. The work planned and described depends wholly on the excellent work of the research assistants in acquiring the data: Matthew Kelly, Laura Wintour, Kirandeep Shetra, Roseanna Bridge, Samir Pathan, Julie Evans, Nicolas Lorenzini, Jennifer O'Connell, Lawson Falshaw, Antonia Blumenstock, Karolina Urbonaite, and Sophie Wallace-Hanlon (UCL research assistants). PF is supported by the NIHR North Thames Applied Research Collaboration. PM is supported by the Biomedical Research Centre at University Hospitals Bristol NHS Foundation Trust and the University of Bristol, England. The views expressed in this publication are those of the author(s) and not necessarily those of the NHS, the National Institute for Health Research, or the Department of Health and Social Care. Finally, we would like to thank the clinical and probation staff involved in the trial for their effort and determination in implementing this new service and research.

\section{Authors' contributions}

$\mathrm{PF}$ is the Chief Investigator. PF, AB, and JY conceived and designed the study. TG, MM, MC, PM, AF, and SP contributed to the study design. MM, MC, $\mathrm{PM}, \mathrm{AF}$, and $\mathrm{SP}$ provided and will continue to provide methodological expertise. AC provided and will continue to provide expertise on interfacing the protocol with the criminal justice system. BB designed and will oversee the health economic analysis and evaluation. JW designed and will perform the statistical analysis. SB is the Project Manager. ES is the Research Manager. TG designed and performed the systematic search strategy and review of the literature. TG drafted the manuscript. All authors participated in the review and revision of the manuscript and approved the final manuscript.

\section{Funding}

The research component of this trial is funded by the National Institute for Health Research (Health Technology Assessment Programme; project reference 14/186/01); the clinical component of this trial is funded by NHS England and NOMS Offender Personality Disorder Team. PF is in part supported by the NIHR Applied Research Collaboration (ARC) North Thames at Barts Health NHS Trust. The views expressed are those of the authors and not necessarily those of the NIHR or the Department of Health and Social Care. None of the funders play an active part in the design of the study, in 
the collection, analysis, and interpretation of the data, or in the writing of this manuscript.

\section{Availability of data and materials} Not applicable.

\section{Ethics approval and consent to participate}

The study protocol was approved by the London - South East Research Ethics Committee (reference number 14/LO/1696) and the National Offender Management Service (reference number 2014-315). Research and development approval will be sought and obtained for each trial site by the relevant NHS Trust and NPS lead in each geographical area.

A key inclusion criterion for trial participants is adequate English language and cognitive capacities to participate in informed consent and group therapy. Potential participants will be provided with participant information sheets before providing consent. Each site's lead MBT clinician will assess potential participants' capacity to provide informed consent and will countersign the consent form if satisfied.

\section{Consent for publication}

There are no details on individuals reported within this manuscript.

\section{Competing interests}

The authors declare that they have no competing interests.

\section{Author details}

${ }^{1}$ Research Department of Clinical, Educational and Health Psychology, University College London, London, UK. ${ }^{2}$ Anna Freud National Centre for Children and Families, London, UK. ${ }^{3}$ Portman Clinic, Tavistock and Portman NHS Foundation Trust, London, UK. ${ }^{4}$ Institute of Mental Health, University of Nottingham, Nottingham, UK. ${ }^{5}$ Centre for Academic Mental Health, Population Health Sciences Department, Bristol Medical School, University of Bristol, Bristol, UK. ${ }^{6}$ Centre for Mental Health, Imperial College, London, UK. ${ }^{7}$ School of Law, Royal Holloway, University of London, London, UK. ${ }^{8}$ Institute of Psychiatry, Psychology and Neuroscience, King's College London, London, UK. ${ }^{9}$ National Probation Service London Division, London, UK. ${ }^{10}$ Population Health Sciences Institute, Newcastle University, Newcastle upon Tyne, UK.

${ }^{11}$ MRC Biostatistics Unit, University of Cambridge, Cambridge, UK.

${ }^{12}$ Psychology Department, University of Prince Edward Island, Charlottetown, Canada.

Received: 17 July 2020 Accepted: 12 November 2020 Published online: 07 December 2020

\section{References}

1. Alwin N, Blackburn R, Davidson K, Hilton M, Logan C, Shine J. Understanding personality disorder: a report by the British Psychological Society. Leicester: British Psychological Society; 2006.

2. McMurran M, Howard R. Personality, personality disorder and violence: an evidence-based approach. Chichester: Wiley; 2009.

3. Department of Health/National Offender Management Service Offender Personality Disorder Team. The offender personality disorder strategy. London: Department of Health; 2011.

4. Singleton N, Gatward R, Meltzer H. Psychiatric morbidity among prisoners in England and Wales. London: Office for National Statistics; 1998.

5. National Institute for Health and Clinical Excellence. Antisocial personality disorder: treatment, management and prevention. Clinical guideline 77. London: The British Psychological Society and The Royal College of Psychiatrists; 2009

6. American Psychiatric Association. Diagnostic and statistical manual of mental disorders, 5th edn (DSM-5). Washington, DC: American Psychiatric Publishing; 2013.

7. Martin RL, Cloninger CR, Guze SB, Clayton PJ. Mortality in a follow-up of 500 psychiatric outpatients. I total mortality. Arch Gen Psychiatry. 1985;42(1):4754. https://doi.org/10.1001/archpsyc.1985.01790240049005.

8. Black DW, Baumgard CH, Bell SE, Kao C. Death rates in 71 men with antisocial personality disorder. A comparison with general population mortality. Psychosomatics. 1996;37(2):131-6. https://doi.org/10.1016/S00333182(96)71579-7.

9. Odgers CL, Caspi A, Broadbent JM, Dickson N, Hancox RJ, Harrington H, Poulton R, Sears MR, Thomson WM, Moffitt TE. Prediction of differential adult health burden by conduct problem subtypes in males. Arch Gen Psychiatry. 2007;64(4):476-84. https://doi.org/10.1001/archpsyc.64.4.476.

10. Piquero AR, Shepherd I, Shepherd JP, Farrington DP. Impact of offending trajectories on health: disability, hospitalisation and death in middle-aged men in the Cambridge Study in Delinquent Development. Crim Behav Ment Health. 2011;21(3):189-201. https://doi.org/10.1002/cbm.810.

11. Swanson MCJ, Bland RC, Newman SC. Antisocial personality disorders. Acta Psychiatr Scand. 1994;89(s376):63-70. https://doi.org/10.1111/j.1600-0447. 1994.tb05792.x.

12. Goodwin RD, Hamilton SP. Lifetime comorbidity of antisocial personality disorder and anxiety disorders among adults in the community. Psychiatry Res. 2003;117(2):159-66. https://doi.org/10.1016/S0165-1781(02)00320-7.

13. Lenzenweger MF, Lane MC, Loranger AW, Kessler RC. DSM-IV personality disorders in the National Comorbidity Survey Replication. Biol Psychiatry. 2007;62(6):553-64. https://doi.org/10.1016/j.biopsych.2006.09.019.

14. Robins LN, Regier DA. An overview of psychiatric disorders in America. In: Psychiatric disorders in America: the epidemiologic catchment area study. New York: Free Press; 1991. p. 328-66.

15. Compton WM, Conway KP, Stinson FS, Colliver JD, Grant BF. Prevalence, correlates, and comorbidity of DSM-IV antisocial personality syndromes and alcohol and specific drug use disorders in the United States: results from the National Epidemiologic Survey on Alcohol and Related Conditions. J Clin Psychiatry. 2005;66(6):677-85. https://doi.org/10.4088/jcp.v66n0602.

16. Byrne SA, Cherniack MG, Petry NM. Antisocial personality disorder is associated with receipt of physical disability benefits in substance abuse treatment patients. Drug Alcohol Depend. 2013;132(1-2):373-7. https://doi. org/10.1016/j.drugalcdep.2013.01.004.

17. Ogloff JR. Psychopathy/antisocial personality disorder conundrum. Aust N Z J Psychiatry. 2006:40(6-7):519-28. https://doi.org/10.1111/j.1440-1614.2006.01834.x.

18. Pluck G, Brooker C, Blizard R, Moran P. Personality disorder in a probation cohort: demographic, substance misuse and forensic characteristics. Crim Behav Ment Health. 2015;25(5):403-15. https://doi.org/10.1002/cbm.1938.

19. Coid J, Yang M, Roberts A, Ullrich S, Moran P, Bebbington P, Brugha T, Jenkins $\mathrm{R}$, Farrell $\mathrm{M}$, Lewis $\mathrm{G}$, et al. Violence and psychiatric morbidity in a national household population - a report from the British Household Survey. Am J Epidemiol. 2006;164(12):1199-208. https://doi.org/10.1093/aje/kwj339.

20. Yu R, Geddes JR, Fazel S. Personality disorders, violence, and antisocial behavior: a systematic review and meta-regression analysis. J Personal Disord. 2012;26(5):775-92. https://doi.org/10.1521/pedi.2012.26.5.775.

21. Hodgins S, Mednick SA, Brennan PA, Schulsinger F, Engberg M. Mental disorder and crime. Evidence from a Danish birth cohort. Arch Gen Psychiatry. 1996;53(6):489-96. https://doi.org/10.1001/archpsyc.1996. 01830060031004

22. Wormith JS, Olver ME, Stevenson HE, Girard L. The long-term prediction of offender recidivism using diagnostic, personality, and risk/need approaches to offender assessment. Psychol Serv. 2007;4(4):287-305. https://doi.org/10. 1037/1541-1559.4.4.287.

23. Home Office, Department of Health. Managing dangerous people with severe personality disorder: proposals for policy development. London: Home Office; 2002.

24. Wilson HA. Can antisocial personality disorder be treated? A meta-analysis examining the effectiveness of treatment in reducing recidivism for individuals diagnosed with ASPD. Int J Forensic Ment Health. 2014;13(1):3646. https://doi.org/10.1080/14999013.2014.890682.

25. Davidson KM, Tyrer P, Tata P, Cooke D, Gumley A, Ford I, Walker A, Bezlyak $V$, Seivewright $H$, Robertson $H$, et al. Cognitive behaviour therapy for violent men with antisocial personality disorder in the community: an exploratory randomized controlled trial. Psychol Med. 2009;39(4):569-77. https://doi.org/ $10.1017 /$ S0033291708004066

26. Cullen AE, Clarke AY, Kuipers E, Hodgins S, Dean K, Fahy T. A multisite randomized trial of a cognitive skills program for male mentally disordered offenders: violence and antisocial behavior outcomes. J Consult Clin Psychol. 2012;80(6):1114-20. https://doi.org/10.1037/a0030291.

27. Doyle M, Khanna T, Lennox C, Shaw J, Hayes A, Taylor J, Roberts A, Dolan $M$. The effectiveness of an enhanced thinking skills programme in offenders with antisocial personality traits. J Forens Psychiatry Psychol. 2013;24(1):1-15. https://doi.org/10.1080/14789949.2012.752519.

28. Khalifa N, Duggan C, Stoffers J, Huband N, Vollm BA, Ferriter M, Lieb K. Pharmacological interventions for antisocial personality disorder. Cochrane Database Syst Rev. 2010(8):CD007667. https://doi.org/10.1002/14651858. CD007667.pub2. 
29. Vollm B, Gibbon S, Khalifa N, Duggan C, Stoffers J, Huband N, Ferriter M, Lieb K. Cochrane reviews of pharmacological and psychological interventions for antisocial personality disorder (ASPD). Eur Psychiatry. 2010; 25(Suppl 1):90. https://doi.org/10.1016/s0924-9338(10)70090-0.

30. Duggan C, Huband N, Smailagic N, Ferriter M, Adams C. The use of pharmacological treatments for people with personality disorder: a systematic review of randomized controlled trials. Personal Ment Health. 2008;2(3):119-70. https://doi.org/10.1002/pmh.41.

31. Warren F, McGauley G, Norton K, Dolan B, Preedy-Fayers K, Pickering A, Geddes J. Review of treatments for severe personality disorder. London: Home Office Research, Development and Statistics Directorate; 2003.

32. Dunn J. The Emanuel Miller Memorial Lecture 1995. Children's relationships: bridging the divide between cognitive and social development. J Child Psychol Psychiatry. 1996;37(5):507-18. https://doi.org/10.1111/j.1469-7610. 1996.tb01437.x.

33. Dunn J, Brophy M. Communication relationships and individual differences in children's understanding of mind. In: Astington JW, Baird JA, editors. Why languages matter for theory of mind. New York: Oxford University Press; 2005. p. 50-69.

34. Dunn J, Cutting AL. Understanding others, and individual differences in friendship interactions in young children. Soc Dev. 1999:8(2):201-19. https:// doi.org/10.1111/1467-9507.00091.

35. Marsh AA, Blair RJ. Deficits in facial affect recognition among antisocial populations: a meta-analysis. Neurosci Biobehav Rev. 2008;32(3):454-65. https://doi.org/10.1016/j.neubiorev.2007.08.003.

36. Tolan PH, Dodge K, Rutter M. Tracking the multiple pathways of parent and family influence on disruptive behavior disorders. In: Tolan PH, Leventhal BL, editors. Disruptive behavior disorders. New York: Springer; 2013. p. 161-91.

37. Mize J, Pettit GS. Social information processing and the development of conduct problems in children and adolescents: looking beneath the surface. In: Sharp C, Fonagy P, Goodyer I, editors. Social cognition and developmental psychopathology. Oxford: Oxford University Press; 2008. p. $141-74$

38. McGauley G, Ferris S, Marin-Avellan L, Fonagy P. The Index Offence Representation Scales; a predictive clinical tool in the management of dangerous, violent patients with personality disorder? Crim Behav Ment Health. 2013;23(4):274-89. https://doi.org/10.1002/cbm.1889.

39. Taubner S, White LO, Zimmermann J, Fonagy P, Nolte T. Attachment-related mentalization moderates the relationship between psychopathic traits and proactive aggression in adolescence. J Abnorm Child Psychol. 2013;41(6): 929-38. https://doi.org/10.1007/s10802-013-9736-X.

40. Vernberg EM, Nelson TD, Fonagy P, Twemlow SW. Victimization, aggression, and visits to the school nurse for somatic complaints, illnesses, and physical injuries. Pediatrics. 2011;127(5):842-8. https://doi.org/10.1542/peds.2009-3415.

41. Hill J, Fonagy $P$, Lancaster G, Broyden N. Aggression and intentionality in narrative responses to conflict and distress story stems: an investigation of boys with disruptive behaviour problems. Attach Hum Dev. 2007:9(3):22337. https://doi.org/10.1080/14616730701453861.

42. Twemlow SW, Fonagy P, Sacco FC. The role of the bystander in the social architecture of bullying and violence in schools and communities. Ann N Y Acad Sci. 2004;1036:215-32. https://doi.org/10. 1196/annals.1330.014.

43. Hill-Smith AJ, Hugo P, Hughes $P$, Fonagy $P$, Hartman D. Adolescents murderers: abuse and adversity in childhood. J Adolesc. 2002;25(2):221-30. https://doi.org/10.1006/jado.2002.0462.

44. Dolan M, Fullam R. Theory of mind and mentalizing ability in antisocial personality disorders with and without psychopathy. Psychol Med. 2004; 34(6):1093-102. https://doi.org/10.1017/S0033291704002028.

45. Newbury-Helps J, Feigenbaum J, Fonagy P. Offenders with antisocial personality disorder display more impairments in mentalizing. J Personal Disord. 2017;31(2):232-55. https://doi.org/10.1521/pedi_2016_30_246.

46. Blair RJ. Neurocognitive models of aggression, the antisocial personality disorders, and psychopathy. J Neurol Neurosurg Psychiatry. 2001;71(6):72731. https://doi.org/10.1136/jnnp.71.6.727

47. Shamay-Tsoory SG, Harari H, Aharon-Peretz J, Levkovitz Y. The role of the orbitofrontal cortex in affective theory of mind deficits in criminal offenders with psychopathic tendencies. Cortex. 2010;46(5):668-77. https:/doi.org/10.1016/j.cortex. 2009.04.008.

48. Fonagy P. Early-life trauma and the psychogenesis and prevention of violence. Ann N Y Acad Sci. 2004;1036:181-200. https://doi.org/10.1196/ annals.1330.012.
49. Fonagy $\mathrm{P}$. Towards a developmental understanding of violence. $\mathrm{Br} \mathrm{J}$ Psychiatry. 2003;183:190-2. https://doi.org/10.1192/bjp.183.3.190.

50. Fonagy $P$, Target M. Attachment and reflective function: their role in selforganization. Dev Psychopathol. 1997;9(4):679-700. https://doi.org/10.1017/ s0954579497001399.

51. Fonagy P. Thinking about thinking: some clinical and theoretical considerations in the treatment of a borderline patient. Int $J$ Psychoanal. 1991;72(Pt 4):639-56.

52. Adshead G, Fonagy P, Sarkar SP. Violence and gun crime. BMJ. 2007; 335(7625):837. https://doi.org/10.1136/bmj.39365.683877.BE.

53. Bateman A, Fonagy P. Comorbid antisocial and borderline personality disorders: mentalization-based treatment. J Clin Psychol. 2008;64(2):181-94. https://doi.org/10.1002/jclp.20451.

54. Fonagy P, Twemlow SW, Vernberg EM, Nelson JM, Dill EJ, Little TD, Sargent JA. A cluster randomized controlled trial of child-focused psychiatric consultation and a school systems-focused intervention to reduce aggression. J Child Psychol Psychiatry. 2009;50(5):607-16. https://doi.org/10. 1111/j.1469-7610.2008.02025.x.

55. Fonagy P, Twemlow SW, Vernberg E, Sacco FC, Little TD. Creating a peaceful school learning environment: the impact of an antibullying program on educational attainment in elementary schools. Med Sci Monit. 2005;11(7):CR317-25.

56. Bateman A, Fonagy P. Impact of clinical severity on outcomes of mentalisation-based treatment for borderline personality disorder. $\mathrm{Br} \mathrm{J}$ Psychiatry. 2013;203(3):221-7. https://doi.org/10.1192/bjp.bp.112.121129.

57. Willmot $\mathrm{P}, \mathrm{McM}$ urran $\mathrm{M}$. The views of male forensic inpatients on how treatment for personality disorder works. J Forensic Psychiatry Psychol. 2013; 24(5):594-609. https://doi.org/10.1080/14789949.2013.823219.

58. Willmot P, McMurran M. An attachment-based model of therapeutic change processes in the treatment of personality disorder among male forensic inpatients. Leg Criminol Psychol. 2016;21(2):390-406. https://doi.org/10. 1111/lcrp.12055.

59. Bateman A, Fonagy P. Mentalization-based treatment for personality disorders: a practical guide. Oxford: Oxford University Press; 2016.

60. Bateman A, Motz A, Yakeley J. Antisocial personality disorder in community and prison settings. In: Bateman A, Fonagy $P$, editors. Handbook of mentalizing in mental health practice. 2nd ed. Washington, DC: American Psychiatric Publishing; 2011. p. 335-49.

61. Bateman A, Fonagy P, Bolton R. Antisocial personality disorder: a mentalizing framework. Focus. 2013;11(2):178-86. https://doi.org/10.1176/ appi.focus.11.2.178.

62. American Psychiatric Association. Diagnostic and statistical manual of mental disorders, 4th edn, text revision (DSM-IV-TR). Washington, DC: American Psychiatric Publishing; 2000.

63. Huband N, McMurran M, Evans C, Duggan C. Social problem-solving plus psychoeducation for adults with personality disorder: pragmatic randomised controlled trial. Br J Psychiatry. 2007;190:307-13. https://doi.org/10.1192/bjp. bp.106.023341.

64. Ball SA, Cobb-Richardson P, Connolly AJ, Bujosa CT, O'Neall TW. Substance abuse and personality disorders in homeless drop-in center clients: symptom severity and psychotherapy retention in a randomized clinical trial. Compr Psychiatry. 2005;46(5): 371-9. https://doi.org/10.1016/j.comppsych.2004.11.003.

65. Berman $\mathrm{AH}$, Bergman $\mathrm{H}$, Palmstierna $\mathrm{T}$, Schlyter F. Evaluation of the Drug Use Disorders Identification Test (DUDIT) in criminal justice and detoxification settings and in a Swedish population sample. Eur Addict Res. 2005;11(1):22-31. https://doi.org/10.1159/000081413.

66. Maffei C, Fossati A, Agostoni I, Barraco A, Bagnato M, Deborah D, Namia C, Novella L, Petrachi M. Interrater reliability and internal consistency of the Structured Clinical Interview for DSM-IV axis II Personality Disorders (SCID-II), version 2.0. J Personal Disord. 1997;11(3): 279-84. https://doi.org/10.1521/pedi.1997.11.3.2799348491.

67. Coccaro EF. Overt aggression scale - modified (OAS-M). In: Rush AJ, First MB, Blacker D, editors. Handbook of psychiatric measures. 2nd ed. Washington, DC: American Psychiatric Publishing; 2008. p. 737-9.

68. Hollander E, Tracy KA, Swann AC, Coccaro EF, McElroy SL, Wozniak P, Sommenille KW, Nemeroff CB. Divalproex in the treatment of impulsive aggression: efficacy in cluster B personality disorders. Neuropsychopharmacology. 2003;28(6):1186-97. https:/doi.org/10.1038/sj.npp.1300153.

69. Roberts C, Roberts SA. Design and analysis of clinical trials with clustering effects due to treatment. Clin Trials. 2005;2(2):152-62. https://doi.org/10. 1191/1740774505cn076oa. 
70. Olver ME, Stockdale KC, Wormith JS. A meta-analysis of predictors of offender treatment attrition and its relationship to recidivism. J Consult Clin Psychol. 2011;79(1):6-21. https://doi.org/10.1037/a0022200.

71. McMurran M, Huband N, Overton E. Non-completion of personality disorder treatments: a systematic review of correlates, consequences, and interventions. Clin Psychol Rev. 2010;30(3):277-87. https://doi.org/10.1016/j. cpr.2009.12.002

72. McCarthy L, Duggan C. Engagement in a medium secure personality disorder service: a comparative study of psychological functioning and offending outcomes. Crim Behav Ment Health. 2010;20(2):112-28. https:// doi.org/10.1002/cbm.758.

73. Byng R, Quinn C, Sheaff R, Samele C, Duggan S, Harrison D, Owens C, Smithson P, Wright C, Annison J, et al. COCOA: care for offenders, continuity of access. Southampton: NIHR Service Delivery and Organisation Programme; 2012.

74. Livingston JD, Nijdam-Jones A, Team PEER. Perceptions of treatment planning in a forensic mental health hospital: a qualitative, participatory action research study. Int J Forensic Ment Health. 2013;12(1):42-52. https:// doi.org/10.1080/14999013.2013.763390.

75. Livingston JD, Desmarais SL, Verdun-Jones S, Parent R, Michalak E, Brink J. Perceptions and experiences of people with mental illness regarding their interactions with police. Int J Law Psychiatry. 2014;37(4):334-40. https://doi. org/10.1016/j.jilp.2014.02.003.

76. Russell D, Hoare ZS, Whitaker R, Whitaker CJ, Russell IT. Generalized method for adaptive randomization in clinical trials. Stat Med. 2011:30(9):922-34. https://doi.org/10.1002/sim.4175.

77. Bateman AW. Mentalization-based treatment adherence and competence scale. London: Anna Freud National Centre for Children and Families; 2018 https://www.annafreud.org/training/mentalization-based-treatment-training/ mbt-adherence-scale. Accessed 9 Nov 2020.

78. Miller TQ, Smith TW, Turner CW, Guijarro ML, Hallet AJ. A meta-analytic review of research on hostility and physical health. Psychol Bull. 1996;119(2): 322-48. https://doi.org/10.1037/0033-2909.119.2.322.

79. Wong JM, Sin NL, Whooley MA. A comparison of Cook-Medley Hostility Subscales and mortality in patients with coronary heart disease: data from the Heart and Soul Study. Psychosom Med. 2014;76(4):311-7. https://doi. org/10.1097/PSY.0000000000000059.

80. White IR, Horton NJ, Carpenter J, Pocock SJ. Strategy for intention to treat analysis in randomised trials with missing outcome data. BMJ. 2011;342:d40. https://doi.org/10.1136/bmj.d40.

81. Steadman HJ, Mulvey EP, Monahan J, Robbins PC, Appelbaum PS, Grisso T, Roth LH, Silver E. Violence by people discharged from acute psychiatric inpatient facilities and by others in the same neighborhoods. Arch Gen Psychiatry. 1998;55(5):393-401. https://doi.org/10.1001/archpsyc.55.5.393.

82. Pailing A, Boon J, Egan V. Personality, the dark triad and violence. Pers Individ Differ. 2014;67:81-6. https://doi.org/10.1016/j.paid.2013.11.018.

83. Spielberger CD, Sydeman SJ, Owen AE, Marsh BJ. Measuring anxiety and anger with the State-Trait Anxiety Inventory (STAI) and the State-Trait Anger Expression Inventory (STAXI). In: The use of psychological testing for treatment planning and outcomes assessment. 2nd ed. Mahwah: Lawrence Erlbaum Associates; 1999. p. 993-1021.

84. Spielberger CD. STAXI-2: State-Trait Anger Expression inventory-2. Professional manual. Odessa: Psychological Assessment Resources; 1999.

85. Rush AJ, First MB, Blacker D. Handbook of psychometric measures. Arlington: American Psychiatric Publishing; 2008.

86. McMurran M. Alcohol-related violence: prevention and treatment. Chichester: Wiley-Blackwell; 2013.

87. Saunders JB, Aasland OG, Babor TF, de la Fuente JR, Grant M. Development of the alcohol use disorders identification test (AUDIT): WHO collaborative project on early detection of persons with harmful alcohol consumption—II. Addiction. 1993;88(6):791-804. https://doi.org/10.1111/j. 1360-0443.1993.tb02093.x.

88. Boles S, Miotto K. Substance abuse and violence: a review of the literature. Aggress Violent Behav. 2003;8(2):155-74.

89. Berman A, Bergman H, Palmstierna T, Schlyter F. DUDIT manual: The Drug Use Disorders Identification Test, version 1.1. Swedish. Stockholm: Karolinska Institutet; 2007.

90. Sansone RA, Wiederman MW, Sansone LA. The Self-Harm Inventory (SHI): development of a scale for identifying self-destructive behaviors and borderline personality disorder. J Clin Psychol. 1998;54(7):973-83. https://doi. org/10.1002/(sici)1097-4679(199811)54:7<973:.:aid-jclp11>3.0.co;2-h.
91. Douglas KS, Lilienfeld SO, Skeem JL, Poythress NG, Edens JF, Patrick CJ. Relation of antisocial and psychopathic traits to suicide-related behavior among offenders. Law Hum Behav. 2008;32(6):511-25. https://doi.org/10. 1007/s10979-007-9122-8.

92. Osman A, Bagge CL, Gutierrez PM, Konick LC, Kopper BA, Barrios FX. The Suicidal Behaviors Questionnaire-Revised (SBQ-R): validation with clinical and nonclinical samples. Assessment. 2001;8(4):443-54. https://doi.org/10. $1177 / 107319110100800409$.

93. Soeteman DI, Verheul R, Busschbach JJ. The burden of disease in personality disorders: diagnosis-specific quality of life. J Personal Disord. 2008;22(3):25968. https://doi.org/10.1521/pedi.2008.22.3.259.

94. Rabin R, de Charro F. EQ-5D: a measure of health status from the EuroQol Group. Ann Med. 2001;33(5):337-43. https://doi.org/10.3109/ 07853890109002087

95. Derogatis LR, Unger R. Symptom checklist-90 - revised. In: Corsini encyclopedia of psychology. Chicago: Wiley; 2010.

96. Krueger R, Derringer J, Markon K, Watson D, Skodol A. The Personality Inventory for DSM-5—Brief Form (PID-5-BF)—Adult. Washington, DC: American Psychiatric Association; 2013.

97. Fonagy $\mathrm{P}$, Luyten $\mathrm{P}$, Moulton-Perkins A, Lee $\mathrm{YW}$, Warren F, Howard S, Ghinai $R$, Fearon P, Lowyck B. Development and validation of a self-report measure of mentalizing: the Reflective Functioning Questionnaire. PLoS One. 2016; 11(7):e0158678. https://doi.org/10.1371/journal.pone.0158678.

98. Tait $L$, Birchwood M, Trower P. A new scale (SES) to measure engagement with community mental health services. J Ment Health. 2002;11(2):191-8. https://doi.org/10.1080/09638230020023570-2

99. National Institute for Health and Care Excellence. Guide to the methods of technology appraisal 2013. London: NICE; 2013.

100. Barrett B, Byford S. Collecting service use data for economic evaluation in DSPD populations: development of the secure facilities service use schedule. Br J Psychiatry. 2007;190(S49):S75-8. https://doi.org/10.1192/bjp. 190.5.s75.

101. Netten A, Knight J, Dennett J, Cooley R, Slight A. A ready reckoner for staff costs in the NHS. Canterbury: Personal Social Services Research Unit, University of Kent; 1998.

102. Department of Health. Reference costs 2011-12. London: Department of Health; 2012.

103. Brookes N, Barrett B, Netten A, Knapp E. Unit costs in criminal justice (UCCJ). Canterbury: University of Kent; 2013.

104. Curtis L. Unit costs of health and social care 2013. Canterbury: Personal Social Services Research Unit, University of Kent; 2013.

105. Thompson SG, Barber JA. How should cost data in pragmatic randomised trials be analysed? BMJ. 2000;320(7243):1197-200. https://doi.org/10.1136/ bmj.320.7243.1197.

106. Barber JA, Thompson SG. Analysis of cost data in randomized trials: an application of the non-parametric bootstrap. Stat Med. 2000;19(23):3219-36. https:/doi.org/10. 1002/1097-0258(20001215)19:23<3219:"Aid-Sim623>3.0.Co;2-P.

107. Devlin N, Shah K, Feng Y, Mulhern B, Van Hout B. Valuing health-related quality of life: an EQ-5D-5L value set for England. London: Office of Health Economics; 2016.

\section{Publisher's Note}

Springer Nature remains neutral with regard to jurisdictional claims in published maps and institutional affiliations.

Ready to submit your research? Choose BMC and benefit from:

- fast, convenient online submission

- thorough peer review by experienced researchers in your field

- rapid publication on acceptance

- support for research data, including large and complex data types

- gold Open Access which fosters wider collaboration and increased citations

- maximum visibility for your research: over $100 \mathrm{M}$ website views per year

At $\mathrm{BMC}$, research is always in progress.

Learn more biomedcentral.com/submission 